\title{
Using Interactive Virtual Characters in Social Neuroscience
}

\author{
Joanna Hale* \\ Institute of Cognitive Neuroscience \\ University College London
}

\author{
Xueni $\mathrm{Pan}^{\dagger}$ \\ Institute of Cognitive Neuroscience \\ University College London
}

\author{
Antonia F. de C. Hamilton ${ }^{+}$ \\ Institute of Cognitive Neuroscience \\ University College London
}

\begin{abstract}
In recent years, the use of Virtual Characters in experimental studies has opened new research avenues in social neuroscience. In this paper, we present the design, implementation, and preliminary results of two case studies exploring different types of social cognition using interactive Virtual Characters animated with motion captured data.
\end{abstract}

Keywords: Virtual Characters, Social Neuroscience, Motion Capture

\section{INTRODUCTION}

Recent advances in the technical ability to build realistic and interactive Virtual Environments (VEs) have allowed neuroscientists to study social cognition and behavior in virtual reality. This is particularly useful in the study of social neuroscience, where the physical appearance and motion of Virtual Characters (VCs) can be fully controlled [1]. The aim of the present two studies was to test what interactive features of VCs are needed to study social cognition in controlled but realistic contexts.

Previous studies suggest that human participants respond towards Virtual Characters (VCs) as if they were real social entities: they keep a polite distance from VCs who make eye contact [2] and display appropriate body movements while interacting with a VC [3]. Very simple VCs have been used to study perspective taking and social exclusion $[4,5]$. More realistic VCs were used to examine social gaze during functional magnetic resonance imaging (fMRI) [6]. VCs which engage in verbal interaction have also been used to study social factors such as rapport [7], persuasion [8], and pro-social emotions [9].

In the following we present two studies which explore how VCs can be used to examine human imitation behavior, and test what interactive features are useful in this regard. Study 1 tests if participants respond faster when cued by a congruent VC movement than when cued by an incongruent $\mathrm{VC}$ movement, building on [10]. Study 2 investigates which aspects of avatar appearance and mimicry behavior influence both implicit and explicit trust. In both studies the participants were motion tracked in real-time. Both studies implemented interactive VR with WorldViz Vizard, and the motion capture was conducted with Polhemus magnetic motion sensors. The VCs were animated in Autodesk motion builder with either real-time streamed or prerecorded data.

\section{Study 1: Congruency Effect in Drumming}

This study tests whether a participant's actions in a drumming

\footnotetext{
* jo.hale.13@ucl.ac.uk

$\dagger$ s.pan@cs.ucl.ac.uk

+a.hamilton@ucl.ac.uk
}

IEEE Virtual Reality Conference 2015

23 - 27 March, Arles, France

978-1-4799-1727-3/15/\$31.00 @2015 IEEE sequence are influenced by a VC's action sequence. In this task, participants take turns with a VC to play short rhythms consists of three beats on three drums. As shown in Fig. 1(a) and (b), participants sit at a table opposite the VC, the participant and VC each have three drums on their table. A virtual iPad provides instructions to the participant. Participants are fitted with two trackers: one on the dominant hand (only right-handed participants were recruited), which they use to perform the drum tapping, and the other one on their forehead. For each trial, first the VC taps three-beat sequence (e.g., "1-3-2") with animations created from a pre-recorded motion capture session. Then the participant is instructed via the virtual iPad to perform their own three-beat sequence. Unbeknownst to the participant, these sequences can be congruent to the action of the VC (e.g. "1-3-2") or incongruent (e.g. "3-1-1"). Note that congruent sequences allow the participant to mirror the VCs actions. During the participant's response period, the VC actively watches the participant, turning her head to follow the participant's head motion. This is implemented with the motion tracker on the participant's head. Participants are instructed that they are taking turns with the VC to play sequences, and must response to their own sequence as fast and accurately as possible. They receive positive (iPad turns green) or negative (the iPad turns red and gives a "beep" error sound) feedback on each trial.

A pilot study was conducted with 22 participants. Each completed three blocks of trials with the VC, and each block contains 48 trials, half congruent and half incongruent, presented in a pseudorandom order. In total for each participant 144 trials (72 congruent and 72 incongruent) were conducted, and only the correct trials were included in the data analysis. The reaction time on each trial was taken as the time from appearance of the cue on the virtual iPad until the time the participant touched the first drum. The congruency effect was calculated as the mean reaction time to incongruent trials minus the mean reaction time to the congruent trials. Result shows a strong congruency effect $(t(21)=$ $-4.6, p<0.001)$, with a faster response in congruent trials $(M=$ $911 \mathrm{~ms}$, S.E. 83.1) than the incongruent trials $(M=941 \mathrm{~ms}$, S.E. 83.4). This demonstrates that VC actions can elicit imitative behavior, even in a minimally interactive context. This study will serve as a baseline for our future studies that will manipulate the appearance or behavior of the $\mathrm{VC}$, and contrast social with nonsocial action cues. The use of Virtual Reality provides essential flexibility and repeatability in these studies.

\section{Study 2: VCs Social Mimicry in Real Time}

We conducted two experiments investigating implicit and explicit trust towards VCs. In each experiment participants interacted with two female VCs which differed in appearance, voice, and nonverbal behavior (all counterbalanced). The VCs were displayed on a projector screen in a virtual extension of the laboratory (Figure 1d). One VC always mimicked the participant's movement, whereas the other moved noncontingently [11]. When a mimicking VC was displayed, the motion trackers on the participant's head and chest (Figure 1c) fed position and rotation data to animate an avatar in real-time. The avatar movement was mirrored and delayed by $4 \mathrm{~s}$, then applied to the $\mathrm{VC}$ to generate social mimicry. When a non-mimicking $\mathrm{VC}$ 


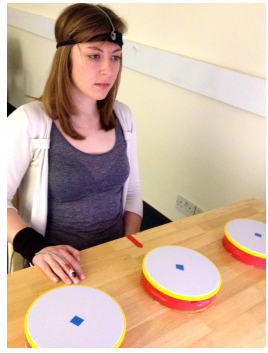

(a)

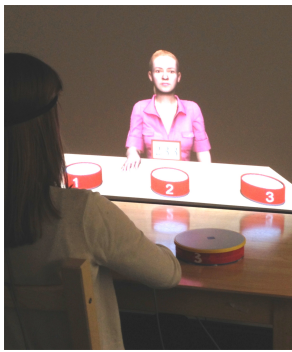

(b)

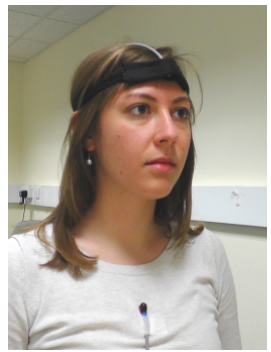

(c)

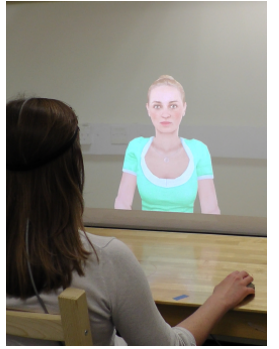

(d)

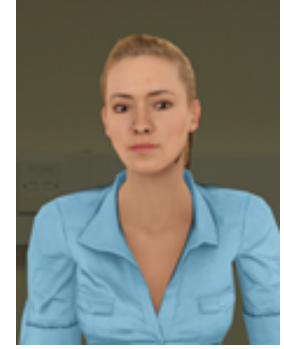

(e)

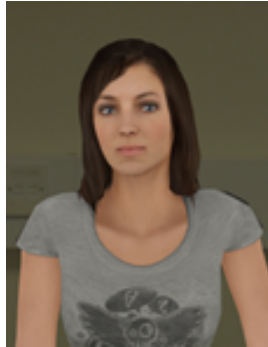

(f)

Figure 1: Figure 1. Using VCs in the study of social neuroscience (a \& b) The drumming paradigm (c, d, e, \& f) mimicry VC

was displayed, pre-recorded motion from a pilot participant was used to animate the $\mathrm{VC}$ in a realistic but non-contingent fashion. Both VCs blinked at suitable intervals and moved their jaw according to speech amplitude.

In the first experiment, 36 female participants listened to each VC deliver two persuasive speeches with accompanying arm gestures and facial expressions (smiles, frowns and eyebrowraising). After each speech, the participant rated agreement on a continuous scale (strongly disagree - strongly agree) with five statements about rapport and five statements about trust towards the VC. They played ten rounds of an economic investment game with each VC, to measure implicit trust in terms of the average amount invested across trials [12]. We analyzed data from 27 participants who did not detect mimicry. The effects of mimicry, appearance and voice on ratings or investment were not significant. Ratings of trust and investment in the economic game were not correlated, suggested a distinction between implicit and explicit trust.

In the second experiment we changed one of the VC appearances to be more distinctive. Twenty-seven participants took five turns with each VC to describe photographs. The VC descriptions were pre-recorded. The participant rated rapport and trust statements as before, and played five rounds of the economic investment game. We analyzed data from 17 participants who did not detect mimicry. There was no significant correlation between ratings of trust and investment in each $\mathrm{VC}$, which replicates our previous finding. There was a significant interaction between mimicry and the mimicking VC's appearance on participants' ratings of trust $\left(F(1,15)=8.2, p=.01, \eta_{p}{ }^{2}=.35\right)$ and rapport $\left(F(1,15)=18.4, p=.001, \eta_{p}{ }^{2}=.55\right)$. These effects were driven by appearance, with participants rating higher rapport and trust towards character A (Fig. 1e) than character B (Fig. 1f). The same effect was not significant for investment $(F(1,15)=3.9, p=.07$, $\left.\eta_{p}{ }^{2}=.21\right)$. There was no significant correlation between ratings of trust and investment in each $\mathrm{VC}$, which replicates our previous finding. Together, these results suggest VC appearance influences explicit feelings of trust more than implicit trust behavior.

\section{Discussion}

We have presented two lines of research to illustrate how VCs can be used in social neuroscience. In Study 1, participants responded faster following congruent $\mathrm{VC}$ movements than following incongruent ones. This extends previous studies of finger movements without VCs [10] by showing that the congruency effect occurs in response to extended sequences of actions performed by another person in a social context. In Study 2, we found that mimicry by a VC can be implemented with acceptable detection rates (26\% for experiment 1 and $38 \%$ for experiment 2 ), but does not influence either explicit or implicit trust. Explicit ratings were influenced by subtle differences in VC appearance, and implicit ratings were unrelated to explicit ratings.

These studies provide a useful starting point for examining social neuroscience with VCs. They show that it is feasible to implement controlled experiments with VCs, and that participants respond well. However, factors including VC appearance must be carefully controlled, and better implicit measures of participant's social response to VCs should be developed. In future, we plan to use these paradigms to test current theories of human social interaction, and to implement VCs in conjunction with neuroimaging methods including fMRI and near-infrared spectroscopy. This will provide realistic, well-controlled social interactions in relation to measures of brain function.

\section{REFERENCES}

[1] J. N. Bailenson, A. C. Beall, J. Loomis, J. Blascovich, and M. Turk, "Transformed social interaction: Decoupling representation from behavior and form in collaborative virtual environments," PRESENCE: Teleoperators and Virtual Environments, vol. 13, pp. 428-441, 2004.

[2] J. N. Bailenson, J. Blascovich, A. C. Beall, and J. M. Loomis, "Equilibrium theory revisited: Mutual gaze and personal space in virtual environments," Presence: Teleoperators and virtual environments, vol. 10, pp. 583-598, 2001.

[3] X. Pan, M. Gillies, and M. Slater, "Male bodily responses during an interaction with a virtual woman," in Intelligent Virtual Agents, 2008, pp. 89-96.

[4] N. David, B. Bewernick, M. Cohen, A. Newen, S. Lux, G. Fink, et al., "Neural representations of self versus other: visual-spatial perspective taking and agency in a virtual ball-tossing game," Cognitive Neuroscience, Journal of, vol. 18, pp. 898-910, 2006.

[5] N. I. Eisenberger, M. D. Lieberman, and K. D. Williams, "Does rejection hurt? An fMRI study of social exclusion," Science, vol. 302, pp. 290-292, 2003.

[6] L. Schilbach, M. Wilms, S. B. Eickhoff, S. Romanzetti, R. Tepest, G. Bente, et al., "Minds made for sharing: initiating joint attention recruits reward-related neurocircuitry," Journal of Cognitive Neuroscience, vol. 22, pp. 2702-2715, 2010.

[7] J. Gratch, N. Wang, J. Gerten, E. Fast, and R. Duffy, "Creating rapport with virtual agents," in Intelligent Virtual Agents, 2007, pp. 125-138.

[8] R. E. Guadagno, J. Blascovich, J. N. Bailenson, and C. Mccall, "Virtual humans and persuasion: The effects of agency and behavioral realism," Media Psychology, vol. 10, pp. 1-22, 2007.

[9] O. Gillath, C. McCall, P. R. Shaver, and J. Blascovich, "What can virtual reality teach us about prosocial tendencies in real and virtual environments?," Media Psychology, vol. 11, pp. 259-282, 2008.

[10] M. Brass, H. Bekkering, A. Wohlschläger, and W. Prinz, "Compatibility between observed and executed finger movements: comparing symbolic, spatial, and imitative cues," Brain and cognition, vol. 44, pp. 124-143, 2000.

[11] J. N. Bailenson and N. Yee, "Digital chameleons automatic assimilation of nonverbal gestures in immersive virtual environments," Psychological science, vol. 16, pp. 814-819, 2005.

[12] J. Berg, J. Dickhaut, and K. McCabe, "Trust, reciprocity, and social history," Games and economic behavior, vol. 10, pp. 122-142, 1995. 